\title{
Erectile dysfunction and cancer: current perspective
}

\author{
Renu Madan', Chinna Babu Dracham², Divya Khosla', Shikha Goyal', Arun Kumar Yadav \\ ${ }^{1}$ Department of Radiotherapy and Oncology, Postgraduate Institute of Medical Education and Research (PGIMER), Chandigarh, India \\ ${ }^{2}$ Department of Radiation Oncology, Queen's NRI Hospital, Visakhapatnam, India
}

Received: June 3, 2020

Revised: July 19, 2020

Accepted: July 30, 2020

Correspondence:

Chinna Babu Dracham

Department of Radiation Oncology,

Queen's NRI Hospital,

Seethammadhara, Gurudwara lane,

Visakhapatnam 530013, India

Tel: +91-9855803437

E-mail:chinnababudraksham@gmail.com

ORCID:

https://orcid.org/0000-0002-8921-0957
Erectile dysfunction (ED) is one of the major but underreported concerns in cancer patients and survivors. It can lead to depression, lack of intimacy between the couple, and impaired quality of life. The causes of erectile dysfunction are psychological distress and endocrinal dysfunction caused by cancer itself or side effect of anticancer treatment like surgery, radiotherapy, chemotherapy and hormonal therapy. The degree of ED depends on age, pre-cancer or pre-treatment potency level, comorbidities, type of cancer and its treatment. Treatment options available for ED are various pharmacotherapies, mechanical devices, penile implants, or reconstructive surgeries. A complete evaluation of sexual functioning should be done before starting anticancer therapy. Management should be individualized and couple counseling should be an integral part of the anticancer treatment.

Keywords: Erectile dysfunction, Neoplasm, Surgery, Radiotherapy

\section{Introduction}

Cancer diagnosis and its management lead to physical and emotional distress in patients. Sexual dysfunction is one of the common problems encountered in these patients which can occur due to direct or indirect pathways. Sexual dysfunction can be in the form of erectile dysfunction (ED) and ejaculatory dysfunction such as decreased or absent semen. Chemotherapy, radiation, hormonal therapy and surgery all can lead to erectile dysfunction. ED has been widely described after prostate cancer treatment. Incidence of post-treatment ED in prostate cancer can vary from $24 \%$ (brachytherapy alone), 40\% (brachytherapy plus external beam radiotherapy [EBRT]), 45\% (EBRT alone), 66\% (nerve-sparing radical prostatectomy [RP]), 75\% (non-nerve sparing RP), and 87\% for cryosurgery [1]. Recently in a population based study patient reported sexual outcome after different types of prostate cancer treatment were reported [2]. Sexual dysfunction after treatment was related to pre-treatment potency, age and type of treatment. No difference in sexual function score was noted in EBRT and brachytherapy at 2 years. Compared with EBRT alone, EBRT with androgen deprivation therapy (ADT) and RP (with or without nerve sparing) was associated with higher incidence of sexual dysfunction. Incidence of ED in cancer patients has been shown in Table 1.

Besides disease control, restoration of quality of life $(\mathrm{QoL})$ is also very important due to increase number of cancer survivors. Evaluating ED using valid questionnaires and management of ED is one of the important aspects of $\mathrm{OoL}$ which is particularly important in developing countries where people are not comfortable discussing about their sexual issues. Age and degree of ED before the diagnosis and mode of treatment play an important role in predicting the post-treatment ED. For this review, we searched PubMed data using words "cancer related erectile dysfunction", "erectile dysfunction in cancer", "sexual dysfunction in genitourinary malignancies," and "psychosocial outcome of cancer survivors". All studies reporting erectile dysfunction in cancer patients and survivors were reviewed. Around 60 studies were screened and data from 54 studies that reported sexual dysfunction or erectile dysfunction in cancer was included in the review.

Copyright (C) 2020 The Korean Society for Radiation Oncology

This is an Open Access article distributed under the terms of the Creative Commons Attribution Non-Commercial License (http://creativecommons.org/licenses/by-nc/4.0/) which permits unrestricted non-commercial use, distribution, and reproduction in any medium, provided the original work is properly cited. 


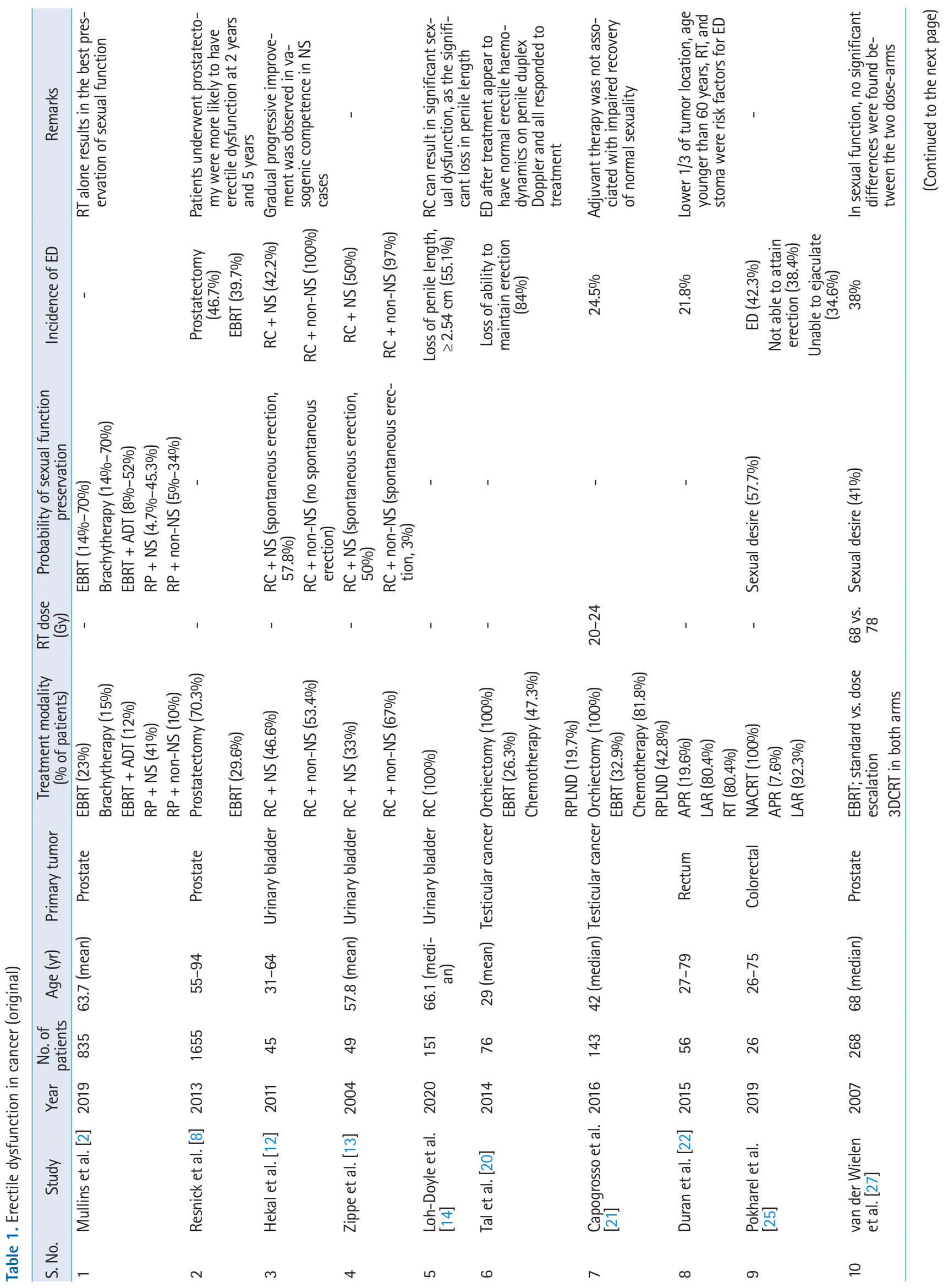




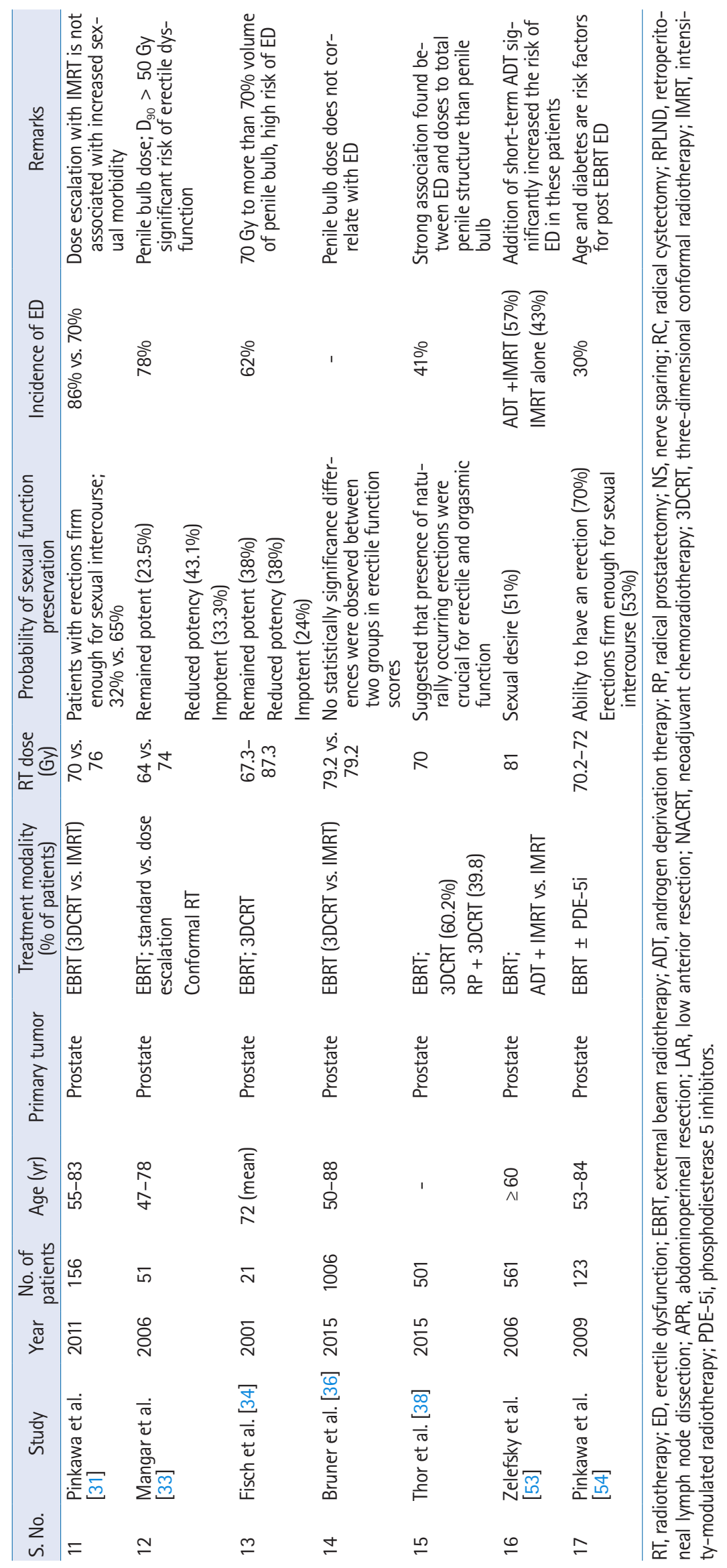




\section{Psychological and emotional impact of ED}

The loss of erectile ability leads to depressive symptoms, lack of sexual satisfaction and general happiness in life. Men who fail to achieve erection tend to lose confidence [3]. Depression and sexual avoidance in turn can affect the intimacy level and relationship of the couple. Studies have reported that ED after curative treatment is one of the common causes of depression in prostate cancer patients.

\section{Mechanism of penile erection}

Blood supply to the penis occurs via branches of external and internal pudendal arteries. Penile innervations are derived from pudendal and cavernous nerves. Pudendal nerve is responsible for somatic motor and sensory nerve supply. Cavernous nerve is a part of autonomic nervous systems and includes sympathetic and parasympathetic fibres. It runs in crus and corpora of the penis and regulates the blood flow during erection and flaccid state [4].

An erection begins with sensory and mental stimulation. Sexual arousal leads to relaxation of smooth muscles of the penile arteries which increases the blood flow to penis. This leads to rigidity and thus erection of the penis. While returning to flaccid state, penile muscles contract and increase the venous outflow and decrease the length and girth of the penis. The ability to attain and sustain erection depends on integrity of vascular supply and nerve supply.

\section{Causes of ED in Cancer}

Causes of ED in cancer patient are follows: (1) emotional, physical and financial stress; (2) pain, anxiety and disturbed body image; (3) damage to penile nerve and vessels during surgery, radiotherapy (RT) and chemotherapy; (4) ADT; (5) penectomy for cancer penis; Nitric oxide (NO) pathway plays an important role in achieving erection. NO is the principle agent responsible for relaxation of penile smooth muscles and thus erection. Radiation leads to damage and inflammation of cavernous nerves, leading to decreased production of nitric oxide synthase and NO which subsequently leads to ED [5]; Fibrotic changes in blood vessels and atrophy of corpora cavernosa due to surgery and RT lead to reduced blood flow in erectile chamber, making it less expansile for blood accumulation during erection.

\section{ED in surgery}

Prostate cancer is one of the most common cancers in males worldwide. Due to widespread use of prostate-specific antigen (PSA), increased numbers of young and healthy men are diagnosed with prostate cancer as compared to past. RP leads to excellent survival and is one of the established treatments for early stage prostate cancer. With excellent disease control, potential side effects of surgery are a growing concern out of which ED is one of the important focuses [6]. Wide range of ED (14\%-90\%) has been reported after RP due to methodological differences in various studies [7]. Resnick et al. [8] published data from the Prostate Cancer Outcomes Study (PCOS) comprising of 1,164 patients who underwent prostatectomy and 491 patients who received RT. These patients were followed up for 15 years. Incidence of ED at 2 years (78.8\% vs. 60.8\%) and 5 years (75.7\% vs. 71.9\%) was higher in surgery as compared to RT. However, the difference was not significant at 15 years. It has been thought that new surgery techniques, i.e., robotic surgery may reduce other side effects, its role in reducing ED is not clear. Nerve sparing surgery in prostate cancer may reduce the incidence of ED. Despite the sparing of cavernous nerve in most of the prostate surgery, it tends to get some sort of bruising and trauma during the surgery which can take up to 2 years to heal. During this time period, men may have difficulty in achieving natural erection which can lead to degeneration of penile tissue and structural alterations [9]. Thus only 9\%-40\% patients resume their sexual function after surgery $[10,11]$. Hekal et al. [12] noted veno-occlusive dysfunction after nerve sparing and non-nerve sparing surgery, which was significantly improved 1 year after nerve-sparing group and spontaneous erections returned after 12 months.

ED can be commonly seen after surgery for bladder cancer. Zippe et al. [13] followed 49 preoperatively sexually active men for 47 months who underwent radical cystectomy $(\mathrm{RC})$ for bladder cancer. At least some degree of ED was found in $86 \%$ of the patients after surgery.

Perception of reduced penile length has been seen after RP and RC. Loh-Doyle et al. [14] analysed sexual function in 151 patients who underwent RC for bladder cancer and observed that 55\% patients perceived loss in penile length and out of the 55\% patients, approximately half of them reported a shortening of 1 inch or more. Erectile dysfunction has also been seen after transurethral resection of bladder tumor (TURBT) and after urinary diversion procedure due to presence of a stoma and sense of disturbed body image [15]. Surgery for penile cancer can also significantly jeopardise sexual function. Scarberry et al. [16] found that after partial penectomy half of the patients can still have normal erection. Gulino et al. [17] reported that after 6 months of conservative surgery (glansectomy) for localized penile cancer, $73 \%$ had spontaneous rigid erections.

Testicular cancer is commonly seen in young patients and thus determining sexual function is even more important. After treatment of testicular cancer, $12 \%-40 \%$ of patients can have ED due to hormonal imbalance including low testosterone, orchidectomy 
or retroperitoneal lymph node dissection $[18,19]$. Tal et al. [20] reported that $84 \%$ patients had loss of sustained erection after treatment although 24\% patients had transient ED even before the diagnosis. In one study it was found that out of every four patients, one patient develops ED, out of which $10 \%$ will have severe ED [21].

Colorectal cancer (CRC) is another common malignancy in males. Surgery for these cancers may also impair sexual function due to hypogastric and autonomic nerve injury [22]. After CRC surgery, sexual dysfunction can range from $10 \%$ to $50 \%$, depending on the assessments used $[23,24]$. In one study, $18 \%$ patients had decreased ejaculation and erection after abdomino-pelvic resection (APR) or low anterior resection (LAR) [22]. A recent study observed that 11 out of 20 patients (42.3\%) had sexual dysfunction after APR/LAR for colorectal cancer [25].

\section{Radiotherapy-induced ED}

Radiotherapy-induced erectile dysfunction (RIED) occurs due to higher dose to penile bulb, crura and neurovascular bundle especially in bladder and prostate cancer. Incidence of RIED has been mentioned above. RIED generally take over 1 year to develop, gradually increases and becomes constant after 3 years [26]. It depends on pre-treatment potency level, RT dose to the penile bulb and use of other concomitant treatment modalities. In a dose escalation study of prostate cancer, incidence of newly diagnosed ED was reported as $36 \%$ and 38\% at 2 and 3 years post-RT [27]. Recent studies have shown that approximately 30\%-40\% patients develop ED after RT [26] as compared to $60 \%-70 \%$ in earlier studies [28]. In one study, ejaculatory disturbance (decreased or absent semen, pain during ejaculation and hemospermia) have been reported in 2\%-56\% after RT while dissatisfaction with sex life can vary from $25 \%$ to $60 \%$ and lack of sexual desire can vary from $12 \%$ to $58 \%$ of the patients with prostate cancer patients after RT [28]. Decreased intensity of orgasm has also been reported. In one study of 262 prostate cancer patients of age $\leq 60$ years, 73\% patients reported either no or only minimal decline in ED after 2 years of RT as compared to baseline [29]. Over the past few years, there has been decrease in the incidence of adverse effects of RT including ED due to increased use of brachytherapy as compared to the past [30] and newer and sophisticated RT techniques like intensity-modulated radiotherapy (IMRT), image-guided radiotherapy (IGRT), and proton therapy although data is insufficient pertinent to ED. In one study, it was reported that sexual function was more retained at 1 year in IMRT arm (30\%) as compared to three-dimensional conformal radiotherapy (3DCRT) arm (14\%) [31]. Siglin et al. [26] showed that decline in sexual function occur maximum after 2 years of RT and then starts stabilising and occasionally can return to baseline.

\section{Radiotherapy dose}

Till date, there is no definite data to suggest the co-relation of RT dose and ED. Relating ED to RT alone is difficult as multiple other factors also contribute to ED like smoking, diabetes mellitus, hypertension, concomitant treatment, etc. Earlier in a study by RTOG group, Roach et al. [32] observed that median penile bulb dose of $>52.5$ Gy is responsible for high risk of ED. In a dose escalation study for prostate cancer, Mangar et al. [33] reported that risk of impotency was significant higher in those whom penile bulb received 50 Gy or more dose of RT.

Few studies have observed that ED and penile bulb dose show a dose volume relationship. Fisch et al. [34] reported ED in 0\%, 80\%, and $100 \%$ of patients when dose received by $70 \%$ of penile bulb volume $\left(D_{70}\right)$ was 0-40 Gy, 40-70 Gy, and > 70 Gy, respectively. Similarly, Mangar et al. [33] observed that if the dose received by $90 \%$ of penile bulb volume $\left(D_{90}\right)$ is 50 Gy or more, it significantly increases the risk of ED. Wernicke et al. [35] reported that dose received by $30 \%, 45 \%, 60 \%$, and $75 \%$ volume of penile bulb $\left(D_{30}\right.$, $D_{45}, D_{60}$, and $D_{75}$, respectively) are also important parameters to define the risk of $E D$.

QUANTEC (Quantitative Analyses of Normal Tissue Effects in the Clinic) has defined that penile bulb may not be a critical structure for ED but may act as surrogate for yet to identified critical structures for ED and suggested a mean dose of not more than $50 \mathrm{~Gy}$ to $95 \%$ volume of penile bulb. In a recently published preliminary patient reported outcomes in RTOG 0126 trial, at 2 years, no significant difference in sexual function was reported in 3DCRT (high penile bulb dose) or IMRT (lower penile bulb dose) arm [36]. However It is recommended that mean RT dose to penile bulb should be kept $<50 \mathrm{~Gy}$ and it is important to limit the $\mathrm{D}_{70}$ to $70 \mathrm{~Gy}$ and $\mathrm{D}_{90}$ to 50 Gy [37].

Based on the patient reported outcomes, Thor et al. [38] identified three domains of sexual dysfunction in males after radiotherapy for prostate cancer, namely ED, orgasmic dysfunction, and pain. He studied the involvement of various penile structures by radiotherapy in ED and observed that other than penile bulb, RT dose to corpora cavernosa (CC) and total penile structure is also important to maintain intact erectile function.

\section{Chemotherapy and hormonal therapy}

Chemotherapy and hormone therapy also play a role in ED. Several chemotherapeutic agents are known to cause ED by causing neural and vasculature damage. These are mainly cisplatin, vincristin and vinblastin. Wiechno et al. [19] found that out of 269 men with testicular cancer, 40\%, developed ED after platinum based chemotherapy. Similarly 20.8\% (out of 202 men) patients with testicular cancer developed ED after platinum based chemotherapy [39]. ED 
has been also observed after high-dose chemotherapy with melphalan, cyclophosphamide or etoposide along with total body radiotherapy in haematological malignancies [40].

ADT is commonly used in the treatment of prostate cancer which is known to cause lack of sexual interest. Loss of libido usually develops within the first several months, and ED follows. In one study sexual inactivity started 6 months after starting ADT [41]. Couples should be counselled before ADT is started. Recovery of erectile function is possible after discontinuation of short-term ADT. However, recovery may be delayed or incomplete [42]. One study has reported that prostate cancer patients who received ADT, are 23\% more likely to develop depression due to ED [43].

\section{Management of ED}

Age of patient and his sexual partner is one of the most important factors while deciding the treatment plan.

\section{Oral and injectable pharmacotherapy}

Phosphodiesterase-5 inhibitors (PDE-5i)/sildenafil citrate (Viagra) is highly an effective and most commonly used oral agent as a first line therapy used in clinical practice [44]. Injury during surgery may lead to hypoxia in penile tissue. PDE-5i increase the release of NO resulting in smooth muscle relaxation and increased blood flow in corpus cavernosum. Sildenafil has an onset of action time, approximately 30 minutes to 1 hour. Cavernous nerve initiates the required erectile pathway so that PDE-5i can be effective. Thus, the effectiveness of these drugs is limited by severity of cavernous nerve injury. Although PDE-5i is the first line treatment for ED, 30\%-40\% patients do exhibit little or no response to it [45]. Prophylactic role of sildenafil citrate in prostate cancer patients was shown by Zelefsky et al. [46]. Patients were randomized to receive either sildenafil daily $50 \mathrm{mg}$ or placebo during and 6 months after RT. In sildenafil arm, significant improvement in ED was noted at 6, 12, and 24 months [46]. Common side effects of sildenafil are headache, facial flushing, stuffy nose and gastrointestinal upset. PDE-5i use is contraindicated in patients taking nitrates for chest pain. It should be used carefully in those who are on alpha-blockers.

Alprostadil (synthetic form of prostaglandin E1 [PGE1]), phentolamine (vasodilator) or papaverine (smooth muscle dilator) can be used for intracavernosal injection [47]. Combination of the drugs is used if one drug fails. As it is a relatively invasive procedure for the patients, it is generally used as a second line therapy in well counselled and motivated patients once treatment becomes refractory to oral agents. Side effects are related to injection site pain and fibrosis.

\section{Intraurethral PGE1}

Alprostadil (MUSE) suppository is inserted in urethra via a small applicator. It creates a vaso-dilatory effect on penile blood vessels and thus helps in penile erection [48].

\section{Vacuum erection device}

It is a battery powered device and can be used in conjunction with a PDE-5i to help maintain and sustain an erection. It consists of cylinder with a pump and constriction ring. Cylinder and pump create vacuum that helps in penile erection while constriction ring helps in maintain erection. Although it is an inexpensive approach, mechanical failure is a problem. Other problems with this device are infection and penile discomfort.

\section{Penile prosthesis implants}

Inflatable or non-inflatable penile implants is an effective safe and durable treatment option for ED and are generally used once patient becomes refractory to other treatment options. In one study it was reported that only $<5 \%$ men underwent penile implantation after RP [49]. Wilson et al. [50] found that 10- and 15-year revision-free survival was $68.9 \%$ and $59.7 \%$, respectively after first time implant.

\section{Vascular reconstruction}

Vascular reconstruction in the form of arterial vascularisation, venous arterialization or venous stripping improves blood flow to corpora cavernosa and thus helps in achieving and maintaining erection [51]. This procedure is generally reserved for those who do not respond to oral or injectable pharmacological therapy.

\section{Low-intensity extracorporeal shock wave therapy} It is a non-invasive newer technology for the treatment of ED. It acts by inducing localized angiogenesis, and pushing blood to the penis [52]. It is safe and can be used in men with failed medical therapy.

\section{Conclusion}

Cancer diagnosis and its treatment can lead to variable degree of sexual dysfunction in patients ranging from loss of libido to complete loss of erection. As sexual issue is a generally less discussed topic, ED is one of the underreported issues in cancer patients. ED can lead to depression and impaired quality of life. Thus, it is very important to evaluate ED before and after treatment. For men who have ED or in those when ED is predictable, couple counselling should be an essential component of the cancer management. Method of sexual rehabilitation should be individualised for each patient. 


\section{Conflict of Interest}

No potential conflict of interest relevant to this article was reported.

\section{References}

1. Robinson JW, Moritz S, Fung T. Meta-analysis of rates of erectile function after treatment of localized prostate carcinoma. Int J Radiat Oncol Biol Phys 2002;54:1063-8.

2. Mullins BT, Basak R, Broughman JR, Chen RC. Patient-reported sexual quality of life after different types of radical prostatectomy and radiotherapy: analysis of a population-based prospective cohort. Cancer 2019;125:3657-65.

3. Nelson CJ, Mulhall JP, Roth AJ. The association between erectile dysfunction and depressive symptoms in men treated for prostate cancer. J Sex Med 2011;8:560-6.

4. Andersson KE. Erectile physiological and pathophysiological pathways involved in erectile dysfunction. J Urol 2003;170(2 Pt 2):S6-14.

5. Mahmood J, Shamah AA, Creed TM, et al. Radiation-induced erectile dysfunction: recent advances and future directions. Adv Radiat Oncol 2016;1:161-9.

6. Liu C, Lopez DS, Chen M, Wang R. Penile rehabilitation therapy following radical prostatectomy: a meta-analysis. J Sex Med 2017:14:1496-503.

7. Tal R, Alphs HH, Krebs P, Nelson CJ, Mulhall JP. Erectile function recovery rate after radical prostatectomy: a meta-analysis. J Sex Med 2009;6:2538-46.

8. Resnick MJ, Koyama T, Fan KH, et al. Long-term functional outcomes after treatment for localized prostate cancer. N Engl J Med 2013;368:436-45.

9. Mulhall J, Land S, Parker M, Waters WB, Flanigan RC. The use of an erectogenic pharmacotherapy regimen following radical prostatectomy improves recovery of spontaneous erectile function. J Sex Med 2005;2:532-42.

10. Jarow JP, Burnett AL, Geringer AM. Clinical efficacy of sildenafil citrate based on etiology and response to prior treatment. J Urol 1999;162(3 Pt 1):722-5.

11. Mahjoubi B, Mirzaei R, Azizi R, Jafarinia M, Zahedi-Shoolami L. A cross-sectional survey of quality of life in colostomates: a report from Iran. Health Qual Life Outcomes 2012;10:136.

12. Hekal IA, Mosbah A, El-Bahnasawy MS, El-Assmy A, Shaaban A. Penile haemodynamic changes in post-radical cystectomy patients. Int J Androl 2011;34:27-32.

13. Zippe $C D$, Raina $R$, Massanyi $E Z$, et al. Sexual function after male radical cystectomy in a sexually active population. Urology
2004;64:682-6.

14. Loh-Doyle JC, Han J, Ghodoussipour S. Factors associated with patient-reported penile length loss after radical cystoprostatectomy in male patients with bladder cancer. J Sex Med 2020;17:957-63.

15. Krajewski W, Halska U, Poletajew S, et al. Influence of transurethral resection of bladder cancer on sexual function, anxiety, and depression. Adv Exp Med Biol 2018;1116:37-50.

16. Scarberry K, Angermeier KW, Montague D, Campbell S, Wood HM. Outcomes for organ-preserving surgery for penile cancer. Sex Med 2015;3:62-6.

17. Gulino G, Sasso F, Palermo G, et al. Sexual outcomes after organ potency-sparing surgery and glans reconstruction in patients with penile carcinoma. Indian J Urol 2013;29:119-23.

18. Rossen $P$, Pedersen $A F$, Zachariae $R$, von der Maase H. Sexuality and body image in long-term survivors of testicular cancer. Eur J Cancer 2012;48:571-8.

19. Wiechno P, Demkow T, Kubiak K, Sadowska M, Kaminska J. The quality of life and hormonal disturbances in testicular cancer survivors in Cisplatin era. Eur Urol 2007;52:1448-54.

20. Tal R, Stember DS, Logmanieh N, Narus J, Mulhall JP. Erectile dysfunction in men treated for testicular cancer. BJU Int 2014;113:907-10.

21. Capogrosso P, Boeri L, Ferrari $M$, et al. Long-term recovery of normal sexual function in testicular cancer survivors. Asian J Androl 2016;18:85-9.

22. Duran $E_{1}$ Tanriseven M, Ersoz N, et al. Urinary and sexual dysfunction rates and risk factors following rectal cancer surgery. Int J Colorectal Dis 2015;30:1547-55.

23. Eveno $C$, Lamblin A, Mariette $C$, Pocard M. Sexual and urinary dysfunction after proctectomy for rectal cancer. J Visc Surg 2010;147:e21-30.

24. Lange $M M$, van de Velde CJ. Urinary and sexual dysfunction after rectal cancer treatment. Nat Rev Urol 2011;8:51-7.

25. Pokharel N, Katwal G, Adhikari SK. Incidence of the bowel, bladder, and sexual dysfunction following surgery for colorectal malignancy. J Nepal Health Res Counc 2019;17:262-7.

26. Siglin J, Kubicek GJ, Leiby B, Valicenti RK. Time of decline in sexual function after external beam radiotherapy for prostate cancer. Int J Radiat Oncol Biol Phys 2010;76:31-5.

27. van der Wielen GJ, van Putten WL, Incrocci L. Sexual function after three-dimensional conformal radiotherapy for prostate cancer: results from a dose-escalation trial. Int J Radiat Oncol Biol Phys 2007;68:479-84.

28. Incrocci L, Slob AK, Levendag PC. Sexual (dys)function after radiotherapy for prostate cancer: a review. Int J Radiat Oncol Biol Phys 2002;52:681-93. 
29. Hoppe BS, Nichols RC, Henderson RH, et al. Erectile function, incontinence, and other quality of life outcomes following proton therapy for prostate cancer in men 60 years old and younger. Cancer 2012;118:4619-26.

30. Challapalli $A$, Jones $E_{1}$ Harvey $C$, Hellawell GO, Mangar SA. High dose rate prostate brachytherapy: an overview of the rationale, experience and emerging applications in the treatment of prostate cancer. Br J Radiol 2012;85 Spec No 1(Spec Iss 1):S18-27.

31. Pinkawa M, Piroth MD, Holy $R$, et al. Combination of dose escalation with technological advances (intensity-modulated and image-guided radiotherapy) is not associated with increased morbidity for patients with prostate cancer. Strahlenther Onkol 2011;187:479-84.

32. Roach M, Winter K, Michalski JM, et al. Penile bulb dose and impotence after three-dimensional conformal radiotherapy for prostate cancer on RTOG 9406: findings from a prospective, multi-institutional, phase I/II dose-escalation study. Int J Radiat Oncol Biol Phys 2004;60:1351-6.

33. Mangar SA, Sydes MR, Tucker HL, et al. Evaluating the relationship between erectile dysfunction and dose received by the penile bulb: using data from a randomised controlled trial of conformal radiotherapy in prostate cancer (MRC RT01, ISRCTN47772397). Radiother Oncol 2006;80:355-62.

34. Fisch BM, Pickett B, Weinberg V, Roach M. Dose of radiation received by the bulb of the penis correlates with risk of impotence after three-dimensional conformal radiotherapy for prostate cancer. Urology 2001;57:955-9.

35. Wernicke AG, Valicenti R, Dieva K, Houser C, Pequignot E. Radiation dose delivered to the proximal penis as a predictor of the risk of erectile dysfunction after three-dimensional conformal radiotherapy for localized prostate cancer. Int J Radiat Oncol Biol Phys 2004;60:1357-63.

36. Bruner DW, Hunt D, Michalski JM, et al. Preliminary patient-reported outcomes analysis of 3-dimensional radiation therapy versus intensity-modulated radiation therapy on the high-dose arm of the Radiation Therapy Oncology Group (RTOG) 0126 prostate cancer trial. Cancer 2015;121:2422-30.

37. Roach M 3rd, Nam J, Gagliardi G, El Naqa I, Deasy JO, Marks LB. Radiation dose-volume effects and the penile bulb. Int J Radiat Oncol Biol Phys 2010;76(3 Suppl):S130-4.

38. Thor $\mathrm{M}$, Olsson $\mathrm{CE}_{1} \mathrm{Oh} \mathrm{JH}$, et al. Radiation dose to the penile structures and patient-reported sexual dysfunction in long-term prostate cancer survivors. J Sex Med 2015;12:2388-97.

39. Bumbasirevic U, Bojanic N, Pekmezovic T, et al. Health-related quality of life, depression, and sexual function in testicular cancer survivors in a developing country: a Serbian experience. Support Care Cancer 2013;21:757-63.
40. Chatterjee $\mathrm{R}$, Andrews HO, McGarrigle HH, et al. Cavernosal arterial insufficiency is a major component of erectile dysfunction in some recipients of high-dose chemotherapy/chemo-radiotherapy for haematological malignancies. Bone Marrow Transplant 2000;25:1185-9.

41. Walker LM, Santos-Iglesias P, Robinson J. Mood, sexuality, and relational intimacy after starting androgen deprivation therapy: implications for couples. Support Care Cancer 2018;26:3835-42.

42. Li R, Ruckle HC, Schlaifer AE, El-Shafei A, Yu C, Jones JS. The effect of androgen deprivation therapy before salvage whole-gland cryoablation after primary radiation failure in prostate cancer treatment. Urology 2015;85:1137-42.

43. Klaassen Z, Arora K, Wilson SN, et al. Decreasing suicide risk among patients with prostate cancer: implications for depression, erectile dysfunction, and suicidal ideation screening. Urol Oncol 2018;36:60-6.

44. Albaugh JA. Addressing and managing erectile dysfunction after prostatectomy for prostate cancer. Urol Nurs 2010;30:167-177.

45. Munk NE, Knudsen JS, Comerma-Steffensen S, Simonsen U. Systematic review of oral combination therapy for erectile dysfunction when phosphodiesterase type 5 inhibitor monotherapy fails. Sex Med Rev 2019;7:430-41.

46. Zelefsky MJ, Shasha D, Branco RD, et al. Prophylactic sildenafil citrate improves select aspects of sexual function in men treated with radiotherapy for prostate cancer. J Urol 2014;192:868-74.

47. Duncan C, Omran GJ, Teh J, Davis NF, Bolton DM, Lawrentschuk N. Erectile dysfunction: a global review of intracavernosal injectables. World J Urol 2019;37:1007-14.

48. Voznesensky M, Annam K, Kreder KJ. Understanding and managing erectile dysfunction in patients treated for cancer. J Oncol Pract 2016;12:297-304.

49. Bajic P, Patel PM, Nelson MH, et al. Penile prosthesis implantation and timing disparities after radical prostatectomy: results from a statewide claims database. J Sex Med 2020;17:1175-81.

50. Wilson SK, Delk JR, Salem EA, Cleves MA. Long-term survival of inflatable penile prostheses: single surgical group experience with 2,384 first-time implants spanning two decades. J Sex Med 2007;4(4 Pt 1):1074-9.

51. Molodysky E, Liu SP, Huang SJ, Hsu GL. Penile vascular surgery for treating erectile dysfunction: current role and future direction. Arab J Urol 2013;11:254-66.

52. Chung E. Penile prosthesis implant: scientific advances and technological innovations over the last four decades. Transl Androl Urol 2017;6:37-45.

53. Zelefsky MJ, Chan H, Hunt M, Yamada Y, Shippy AM, Amols H. Long-term outcome of high dose intensity modulated radiation therapy for patients with clinically localized prostate cancer. J 
Urol 2006;176(4 Pt 1):1415-9.

54. Pinkawa M, Gagel B, Piroth MD, et al. Erectile dysfunction after external beam radiotherapy for prostate cancer. Eur Urol 2009;55:227-34. 